International Mathematical Forum, Vol. 8, 2013, no. 5, 229 - 235

\title{
On the Banach-Steinhaus Theorem
}

Dinamérico P. Pombo Jr.

Instituto de Matemática

Universidade Federal do Rio de Janeiro

Caixa Postal 68530

21945-970 Rio de Janeiro, RJ Brasil

dpombo@terra.com.br

\begin{abstract}
A Banach-Steinhaus theorem for sets of continuous linear mappings on topological modules which are Baire spaces is proved, and some consequences of it are derived.
\end{abstract}

Mathematics Subject Classification: 46H25, 46S10, 46E10

Keywords: topological modules, Banach-Steinhaus theorem, continuous bilinear mappings

\section{Introduction}

The Banach-Steinhaus theorem, whose first version was established in the fundamental paper [2] (see also [3]), is one of the central principles of Functional Analysis. Since the publication of that work, various authors have been discussing the validity of the Banach-Steinhaus theorem in different contexts and under suitable hypotheses, either in the linear case $[4,7,10,11,14,15,16$, $17,18,19,20,21]$ or in the multilinear case $[6,9]$. A common property valid in all the works just mentioned is that the origin of the base unitary topological ring $R$ under consideration is an adherent point of the multiplicative group $R^{X}$ of invertible elements of $R$; and, moreover, this fact plays an important role in the proofs of the various versions of the theorem.

The purpose of this note is to prove a Banach-Steinhaus theorem in the framework of topological modules over certain unitary topological rings $R$ which holds, in particular, if $0 \in \overline{R^{X}}$, although such condition is not assumed to be satisfied. In addition, applications of our result are presented.

Throughout this note $R$ will denote a metrizable topological ring with a 
non-zero identity element and all topological $R$-modules under consideration will be unitary left topological $R$-modules. Given two topological $R$-modules $E$ and $F, \mathcal{L}(E ; F)$ will denote the additive group of all continuous $R$-linear mappings from $E$ into $F$, which is a unitary $R$-module when $R$ is commutative. A subset $\mathfrak{X}$ of $\mathcal{L}(E ; F)$ is said to be pointwise bounded if the set $\mathfrak{X}(x)=$ $\{u(x) ; u \in \mathfrak{X}\}$ is bounded in $F$ for all $x \in E$.

\section{The main result and applications}

Our main result reads as follows:

Theorem 1. Let $E, F$ be two topological $R$-modules such that $E$ is a Baire space and the product of any neighborhood of 0 in $R$ by any neighborhood of 0 in $E$ is a neighborhood of 0 in $E$. If $\mathfrak{X}$ is a pointwise bounded subset of $\mathcal{L}(E ; F)$, then $\mathfrak{X}$ is equicontinuous.

Proof. Let $\left(W_{n}\right)_{n \in \mathbb{N}}$ be a countable fundamental system of neighborhoods of 0 in $R$, and let $V$ be an arbitrary neighborhood 0 in $F$. Let $V_{1}$ be a closed neighborhood of 0 in $F$ such that $V_{1}-V_{1} \subset V$ and, for each $n \in \mathbb{N}$, put

$$
G_{n}=\left\{x \in E ; W_{n} \mathfrak{X}(x) \subset V_{1}\right\}=\bigcap_{u \in \mathfrak{X}}\left\{x \in E ; W_{n} u(x) \subset V_{1}\right\} .
$$

We claim that $E=\bigcup_{n \in \mathbb{N}} G_{n}$. In fact, let $x \in E$ be arbitrary. Since $\mathfrak{X}(x)$ is bounded, there exists a neighborhood $W$ of 0 in $R$ such that $W \mathfrak{X}(x) \subset V_{1}$. On the other hand, there is an $m \in \mathbb{N}$ so that $W_{m} \subset W$, and hence $x \in G_{m}$. Thus $E=\bigcup_{n \in \mathbb{N}} G_{n}$.

Now, we claim that $G_{n}$ is closed in $E$ for all $n \in \mathbb{N}$. Indeed, let $n \in \mathbb{N}$ be fixed and $u \in \mathfrak{X}$ be arbitrary, and let us show that the set

$$
G_{n}^{(u)}=\left\{x \in E ; W_{n} u(x) \subset V_{1}\right\}
$$

is closed in $E$. For this purpose, take a net $\left(x_{i}\right)_{i \in I}$ in $G_{n}^{(u)}$ converging to an element $x \in E$. We have to show that $x \in G_{n}^{(u)}$. But, for any $\lambda \in W_{n}$, we have that $u\left(\lambda x_{i}\right)=\lambda u\left(x_{i}\right) \in V_{1}$ for all $i \in I$ and, moreover, $\left(\lambda x_{i}\right)_{i \in I}$ converges to $\lambda x$. By the continuity of $u$ and the closedness of $V_{1}$, we conclude that $\lambda u(x) \in V_{1}$; thus $x \in G_{n}^{(u)}$. Consequently, $G_{n}$ is closed in $E$.

Since $E$ is a Baire space, there exists an $m \in \mathbb{N}$ so that $\operatorname{int}\left(G_{m}\right) \neq \varnothing$, and hence there is a non-empty open subset $A$ of $E$ such that $A \subset G_{m}$. Therefore, if $y \in A, U=A+(-A)$ is an open subset of $E$ containing $0=y+(-y)$ and

$$
\mathfrak{X}\left(W_{m} U\right) \subset \mathfrak{X}\left(W_{m} A-W_{m} A\right) \subset \mathfrak{X}\left(W_{m} A\right)-\mathfrak{X}\left(W_{m} A\right) \subset V_{1}-V_{1} \subset V .
$$


Finally, by hypothesis, $W_{m} U$ is a neighborhood of 0 in $E$, and therefore $\mathfrak{X}$ is equicontinuous.

Remark 2. Suppose that $0 \in \overline{R^{X}}$, where $R^{X}$ is the multiplicative group of all invertible elements of $R$. Then the condition concerning product of neighborhoods of 0 , which occurs in Theorem 1 and all the other results of this note (except Lemma 8), is always true. In fact, let $E$ be an arbitrary topological $R$-module, $W$ a neighborhood of 0 in $R$ and $U$ a neighborhood of 0 in $E$. Since $0 \in \overline{R^{X}}$, there is a $\lambda \in R^{X} \cap W$. Thus $W U$ is a neighborhood of 0 in $E$ because $W U \supset \lambda U$ and $\lambda U$ is a neighborhood of 0 in $E$ ([21], Theorem $12.4(1))$.

Remark 3. If $E$ is a topological $R$-module such that the product of any neighborhood of 0 in $R$ by any neighborhood of 0 in $E$ is a neighborhood of 0 in $E$, then the same holds for the quotient topological $R$-module $E / M$, where $M$ is an arbitrary submodule of $E$. In fact, if $\pi: E \rightarrow E / M$ is the canonical surjection, $W$ is a neighborhood of 0 in $R$ and $U$ is a neighborhood of 0 in $E$, then $W \pi(U)(=\pi(W U))$ is a neighborhood of 0 in $E / M$.

In particular, if $E$ is metrizable and complete and $M$ is closed, then $E / M$ is a metrizable complete topological $R$-module for which the above-mentioned property is valid.

Example 4. Let $S$ be a discrete valuation $\operatorname{ring}([1], 1.4)$; then $0 \notin \overline{S^{X}}$. For each integer $n \geq 1$ consider the $S$-module $S^{n}$ endowed with the product topology, which makes $S^{n}$ a metrizable linearly topologized $S$-module (which is complete if $S$ is complete). Then the condition about product of neighborhoods of 0 is satisfied by $S^{n}$. This follows from the observation that if $M$ is the maximal ideal of $S$ and $m, m_{1}, \ldots, m_{n}$ are arbitrary integers $\geq 1$, then

$$
M^{m}\left(M^{m_{1}} \times \cdots \times M^{m_{n}}\right)=M^{m+m_{1}} \times \cdots \times M^{m+m_{n}} .
$$

Corollary 5. Let $E$ and $F$ be as in Theorem 1, with $F$ separated. Let $\left(u_{i}\right)_{i \in I}$ be a pointwise bounded net in $\mathcal{L}(E ; F)$, pointwise convergent to a mapping $u: E \rightarrow F$. Then $u \in \mathcal{L}(E ; F)$. In particular, if $\left(u_{n}\right)_{n \in \mathbb{N}}$ is a sequence in $\mathcal{L}(E ; F)$, pointwise convergent to a mapping $u: E \rightarrow F$, then $u \in \mathcal{L}(E ; F)$.

Proof. Obviously, $u$ is an $R$-linear mapping. Put $\mathfrak{X}=\left\{u_{i} ; i \in I\right\}$. By Theorem $1, \mathfrak{X}$ is equicontinuous, and hence $u \in \mathcal{L}(E ; F)$.

Corollary 6. Let $E$ and $F$ be as in Theorem 1 . Then, for any subset $\mathfrak{X}$ of $\mathcal{L}(E ; F)$, the following conditions are equivalent:

(a) $\mathfrak{X}$ is equicontinuous; 
(b) $\mathfrak{X}$ transforms bounded subsets of $E$ into bounded subset of $F$;

(c) $\mathfrak{X}$ transforms compact subsets of $E$ into bounded subsets of $F$;

(d) $\mathfrak{X}$ is pointwise bounded.

Proof. (a) $\Rightarrow$ (b) follows from Theorem 25.5 of [21], (b) $\Rightarrow$ (c) follows from Theorem 15.4 (2) of [21], (c) $\Rightarrow$ (d) is obvious, and (d) $\Rightarrow$ (a) follows from Theorem 1.

Assume that $R$ is commutative. Let $E, F$ be two arbitrary topological $R$-modules and $\mathcal{M}$ a set of bounded subsets of $E$. Since every element of $\mathcal{L}(E ; F)$ transforms bounded sets into bounded sets, it follows from Lemma 1 of [4] that $\left(\mathcal{L}(E ; F), \tau_{\mathcal{M}}\right)$ is a topological $R$-module, where $\tau_{\mathcal{M}}$ is the topology of $\mathcal{M}$-convergence on $\mathcal{L}(E ; F)$.

Before stating another application of Theorem 1 let us recall that a topological $R$-module $F$ is quasi-complete if every bounded and closed subset of $F$ is complete.

Corollary 7. Assume that $R$ is commutative. Let $E$ be as in Theorem 1 and $\mathcal{M}$ a covering of $E$ by bounded subsets of $E$. If $F$ is a separated quasi-complete topological $R$-module, then $\left(\mathcal{L}(E ; F), \tau_{\mathcal{M}}\right)$ is a separated quasicomplete topological $R$-module. In particular, $\left(\mathcal{L}(E ; F), \tau_{s}\right),\left(\mathcal{L}(E ; F), \tau_{0}\right)$ and $\left(\mathcal{L}(E ; F), \tau_{b}\right)$ are separated quasi-complete topological $R$-modules, where $\tau_{s}$ (resp. $\left.\tau_{0}, \tau_{b}\right)$ is the $R$-module topology of pointwise (resp. compact, bounded) convergence on $\mathcal{L}(E ; F)$.

In order to prove Corollary 7 we shall need a result whose proof is analogous to that of Lemma 18 of [9].

Lemma 8. Assume that $R$ is commutative. Let $E$ be an arbitrary topological $R$-module and $\mathcal{M}$ a covering of $E$ by bounded subsets of $E$, and let $F$ be a separated quasi-complete topological $R$-module. If $\mathfrak{X}$ is an equicontinuous subset of $\mathcal{L}(E ; F)$ which is closed in $\left(\mathcal{L}(E ; F), \tau_{\mathcal{M}}\right)$, then $\mathfrak{X}$ is complete in $\left(\mathcal{L}(E ; F), \tau_{\mathcal{M}}\right)$.

Now, let us turn to the

Proof of Corollary 7. First, $\tau_{\mathcal{M}}$ is separated because $\mathcal{M}$ is a covering of $E$ and $F$ is separated. Let $\mathfrak{X}$ be an arbitrary bounded and closed subset of $\left(\mathcal{L}(E ; F), \tau_{\mathcal{M}}\right)$. Then $\mathfrak{X}$ is pointwise bounded since $\mathcal{M}$ is a covering of $E$, and Theorem 1 ensures the equicontinuity of $\mathfrak{X}$. Therefore, by Lemma $8, \mathfrak{X}$ is complete in $\left(\mathcal{L}(E ; F), \tau_{\mathcal{M}}\right)$, and the proof is concluded.

Proposition 9. Assume that $R$ is commutative. Let $E, F$ be two metrizable topological $R$-modules, with $E$ complete, such that product of any neighborhood of 0 in $R$ by any neighborhood of 0 in $E$ (resp. $F$ ) is a neighborhood of 
0 in $E$ (resp. $F$ ), and let $G$ be an arbitrary topological $R$-module. If $\mathfrak{X}$ is a set of $R$-bilinear mappings from $E \times F$ into $G$ such that, for each $x \in E$, the set $\{y \in F \mapsto u(x, y) \in G ; u \in \mathfrak{X}\}$ is equicontinuous and, for each $y \in F$ and for each $u \in \mathfrak{X}$, the mapping $x \in E \mapsto u(x, y) \in G$ is continuous, then $\mathfrak{X}$ is equicontinuous. In particular, every separately equicontinuous set of $R$-bilinear mappings from $E \times F$ into $G$ is equicontinuous.

Proof. We shall argue as in the classical case ([8], pp. 28-30; [12], pp. 58-60). By the theorem proved in [5], it suffices to establish the equicontinuity of $\mathfrak{X}$ at $(0,0)$. Let $\left(\left(x_{n}, y_{n}\right)\right)_{n \in \mathbb{N}}$ be an arbitrary null sequence in $E \times F$ and consider the set

$$
\mathcal{Y}=\left\{x \in E \mapsto u\left(x, y_{n}\right) \in G ; n \in \mathbb{N}, u \in \mathfrak{X}\right\}
$$

of continuous $R$-linear mappings from $E$ into $G$. We claim that $\mathcal{Y}$ is equicontinuous. By Theorem 1 , it is enough to show that $\mathcal{Y}$ is pointwise bounded. So let $x \in E$ be given and consider the equicontinuous set

$$
\mathcal{Z}=\{y \in F \mapsto u(x, y) \in G ; u \in \mathfrak{X}\}
$$

of $R$-linear mappings from $F$ into $G$. Since $\left(y_{n}\right)_{n \in \mathbb{N}}$ is a null sequence in $F$, the set $\left\{y_{n} ; n \in \mathbb{N}\right\}$ is bounded in $F$, and the equicontinuity of $\mathcal{Z}$ implies the boundedness of $\mathcal{Y}(x)$. Therefore, if $V$ is an arbitrary neighborhood of 0 in $G$, there exists a neighborhood $U$ of 0 in $E$ such that $\mathcal{Y}(U) \subset V$. Consequently, since $x_{n} \in U$ for $n$ bigger than a certain $m \in \mathbb{N}$, we conclude that $u\left(x_{n}, y_{n}\right) \in V$ for $n>m$ and $u \in \mathfrak{X}$. We have just proved that, for each null sequence $\left(\left(x_{n}, y_{n}\right)\right)_{n \in \mathbb{N}}$ in $E \times F$, the sequence $\left(u\left(x_{n}, y_{n}\right)\right)_{n \in \mathbb{N}}$ converges uniformly to 0 for $u \in \mathfrak{X}$. Thus, by (4), p. 172 of [13], $\mathfrak{X}$ is equicontinuous at $(0,0)$, which concludes the proof.

\section{References}

[1] Y. Amice, Les nombres p-adiques, Presses Universitaires de France, 1975 .

[2] S. Banach \& H. Steinhaus, Sur le principe de la condensation des singularités, Fund. Math. 9 (1927), 50-61.

[3] S. Banach, Théorie des Opérations Linéaires, Warszawa, 1932.

[4] N.C. Bernardes Jr. \& D.P. Pombo Jr., Bornological topological modules, Math. Japonica 40 (1994), 455-459. 
[5] N.C. Bernardes Jr., A note on the continuity of multilinear mappings in topological modules, Internat. J. Math. \& Math. Sci. 19 (1996), 205-207.

[6] J. Bochnak \& J. Siciak, Polynomials and multilinear mappings in topological vector spaces, Studia Math. 39 (1971), 59-76.

[7] N. Bourbaki, Sur certains espaces vectoriels topologiques, Ann. Inst. Fourier 2 (1950), 5-16.

[8] N. Bourbaki, Espaces Vectoriels Topologiques, Chapitres III, IV et V, Actualités Scientifiques et Industrielles 1229, Hermann, 1967.

[9] C.F.R. Concordido \& D.P. Pombo Jr., Polynomials and multilinear mappings in topological modules, Rend. Circ. Mat. Palermo 51 (2002), $213-236$.

[10] R.R. Del-Vecchio, D.P. Pombo Jr. \& C.T.M. Vinagre, On the BanachSteinhaus theorem and the closed graph theorem in the context of topological modules, Math. Japonica 52 (2000), 415-423.

[11] J. Dieudonné \& L. Schwartz, La dualité dans les espaces $(\mathcal{F})$ et $(\mathcal{L} \mathcal{F})$, Ann. Inst. Fourier 1 (1949), 61-101.

[12] A. Grothendieck, Espaces Vectoriels Topologiques, Terceira edição, Publicação da Sociedade de Matemática de São Paulo, 1964.

[13] G. Köthe, Topological Vector Spaces I, Grundlehren der mathematischen Wissenschaften 159, Springer-Verlag, 1969.

[14] A.F. Monna, Sur le théorème de Banach-Steinhaus, Indag. Math. 66 (1963), 121-131.

[15] D.P. Pombo Jr., On barrelled topological modules, Internat. J. Math. \& Math. Sci. 19 (1996), 45-52.

[16] J.B. Prolla, Topics in Functional Analysis over Valued Division Rings, Notas de Matemática 89, North-Holland, 1982.

[17] W. Robertson, Completions of topological vector spaces, Proc. London Math. Soc. 8 (1958), 242-257.

[18] J. van Tiel, Espaces localement K-convexes, Indag. Math. 68 (1965), 249-289. 
[19] S. Tomás̆ek, M-barrelled spaces, Comment. Math. Univ. Carolinae 11 (1970), 185-204.

[20] L. Waelbroeck, Topological Vector Spaces and Algebras, Lecture Notes in Mathematics 230, Springer-Verlag, 1971.

[21] S. Warner, Topological Fields, Notas de Matemática 126, North-Holland, 1989.

Received: October, 2012 